\title{
Service Agent-Based Resource Management Using Virtualization for Computational Grid
}

\author{
Sung Ho Jang and Jong Sik Lee \\ School of Computer Science and Engineering \\ Inha University \\ Incheon 402-751, South Korea \\ ho7809@hanmail.net, jslee@inha.ac.kr
}

\begin{abstract}
The key purpose of computational grid is how to allocate grid resources to grid applications effectively. Existing resource management models with centralized architecture like GRAM and nimrod-G broker are liable to generate communication overheads and bottleneck. Therefore, we propose the service agent-based resource management model using virtualization for computational grid. Virtualization and agent-based system applied to our model is able to improve load imbalance of grid broker and reduce communication overheads. Experiment results demonstrate that the service agent-based resource management model decreases $25.8 \%$ more job latencies and $151.6 \%$ more communication messages than the centralized resource management model.
\end{abstract}

Keywords: Grid Computing, Agent-based System, Resource Management.

\section{Introduction}

Computational grid [1], the core field of grid computing, enables us to get the ability to perform high performance computing and large-scale simulation. The key purpose of computational grid is how to allocate grid resources to grid applications with high throughput and low latency effectively. But, the effective allocation of grid resources is not an easy task because distributed grid resources have heterogeneous operating systems and different system performances. Grid resources also have commercial characteristics [2] that grid users pay for grid resource utilization and grid resource providers make profits. And, we need to consider the QoS of the whole grid.

Diverse models like GRAM [3] and nimrod-G resource broker [4] have been developed for the resource management of computational grid. GRAM (Globus Resource Allocation Manager), the core component of globus toolkit, is developed to facilitate processing requests for executing remote application and managing active jobs. Nimrod-G resource broker, a grid application scheduler, is responsible for resource discovery, selection, scheduling, and deployment of computations. But, existing models are based on centralized architecture that deals with communications between grid resources and grid applications. And, these models are unable to satisfy commercial characters and meet the dynamic demand for grid resources due to the 
passive resource transaction mechanism. Also, these models are liable to generate communication overheads and bottleneck by communication messages concentrated to grid broker and too many given tasks of grid broker.

Therefore, this paper proposes the service agent-based resource management model using virtualization in order to solve problems of the centralized resource management model. Agent-based system [5] provides the ability to unify and allocate networked resources in the grid environment and is useful to solve technical problems of distributed resource management. As applying agent-based system and virtualization to our model, we can integrate grid resources of local networks and construct a virtual grid computing system.

This paper is organized as follows. Section 2 proposes the service agent-based resource management model. Section 3 demonstrates the efficiency of our model with experiment results. The conclusion of this paper is in Section 4.

\section{Service Agent-Based Resource Management Model}

This paper proposes the service agent-based resource management model for the effective resource management of computational grid. As shown in fig. 1, the service agent-based resource management model consists of five types of components which are grid user, grid resource provider, grid service agent, central grid broker, and local grid broker.

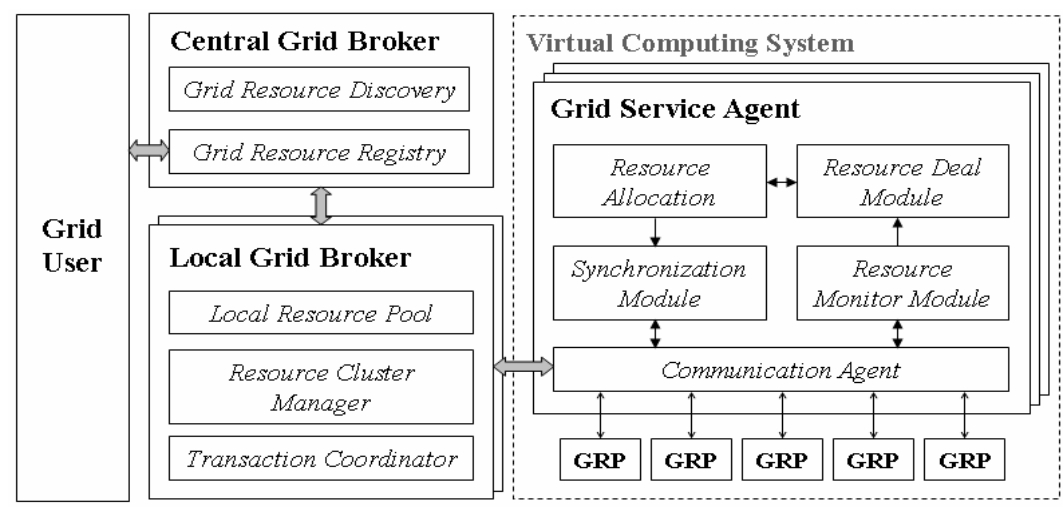

Fig. 1. Service Agent-based Resource Management Model

Roles of each component are as follows. Grid user is in grid application level and uses grid resources to solve its own computing problem as paying for resources. Grid resource provider (GRP) is in grid fabric level and provides commercial grid resources to grid users and makes profits. Central grid broker takes charge of grid resource registry which services the data management of local grid resource group and discovers grid resources suitable for requirements of grid users by data query. Central grid broker also binds grid users with local grid broker of local network of which grid resources satisfy the requirements. 
Local grid broker is composed of local resource pool, resource cluster manager and transaction coordinator. All grid resources connected with local network are stored to local resource pool. Resource cluster manager divides grid resources of this pool into classes and constructs several clusters. Transaction coordinator provides a transaction mechanism by the negotiation of grid user and grid service agent.

Grid service agent, the key component of our model, consists of resource allocation, resource deal module, synchronization module, resource monitor module, and communication agent. Firstly, grid service agent constructs virtual computing system as integrating networked grid resources of a cluster by virtualization techniques [6] such as LUN (Logical Unit Number) mapping and masking. Virtual computing system collects the local network attached computing resources into a large computing system. This logical computing system can be allocated to any grid user with adequate computing performance. It also can solve the physical complexity of grid resources and provide the ability to monitor and control distributed grid resources. Secondly, grid service agent manages metadata about location, owner, and condition of grid resources and improves the monolithic update cycle of metadata as updating and publishing metadata dynamically when the condition of grid resources is changed. Thirdly, grid service agent takes charge of bidding for resource transaction instead of grid resource providers because it costs a great deal for marketing, management and communication if grid resource providers participate in resource transaction in several. Finally, grid service agent prioritizes grid resources of virtual computing system by performance like the processing time and the number of processors.

\section{Experiments and Performance Evaluation}

For the performance evaluation of our model, we implemented it on the DEVS modeling and simulation environment [7] and conducted experiments in comparison with the centralized resource management model of which grid resource providers participate in resource transaction in person.
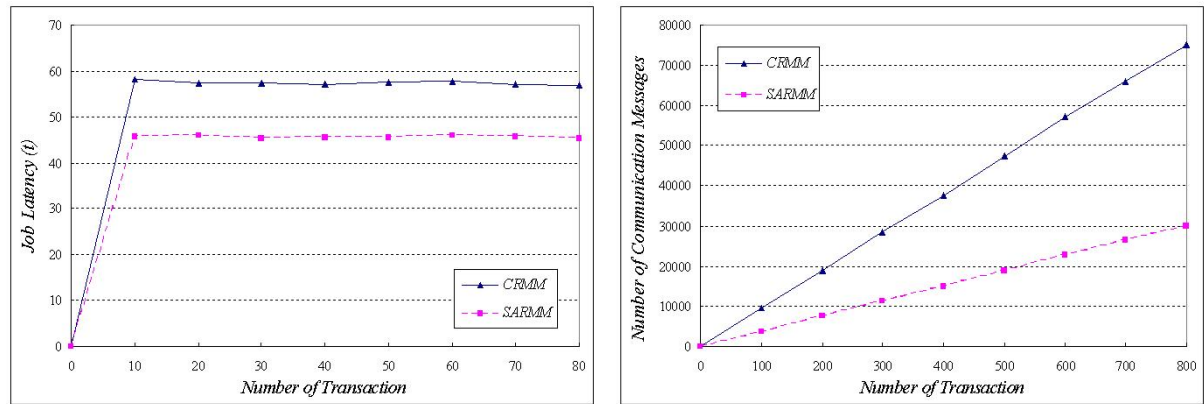

Fig. 2. (a) Comparison of Job Latency (b) Comparison of Communication Messages (Service Agent-based Resource Management Model (SARMM) vs. Centralized Resource Management Model (CRMM)) 
In our experiments, we assumed that grid resources of each model are processors with multi queue and measured the job latency and communication message of each model. Fig. 2 (a) and (b) illustrate variations of job latency and communication messages by the number of transactions. As a result, the decreasing rates of job latency and communication messages for the service agent-based resource management model over the centralized resource management model are $25.8 \%$ and $151.6 \%$. This result demonstrates that our model provides improved QoS with high throughput and reduces communication overheads.

\section{Conclusion}

This paper proposed the service agent-based resource management model to solve problems of existing centralized resource management models in the computational grid environment. We applied virtualization and agent-based system to the service agent-based resource management model in order to improve load imbalance of grid broker and reduce communication overheads. Contrary to the centralized resource management model, our model of which grid service agent takes charge of dealing and monitoring grid resources instead of grid resource providers can generate more resource transactions and profits than existing models. Experiment results demonstrate that the service agent-based resource management model decreases $25.8 \%$ more job latencies and $151.6 \%$ more communication messages than the centralized resource management model.

Acknowledgments. This work is supported by INHA UNIVERSITY Research Grant.

\section{References}

1. Berman, F., Fox, G., Hey, T.: Grid computing: making the global infrastructure a reality. J. Wiley. New York (2003) p

2. Subramoniam, K., Maheswaran, M., Toulouse, M.: Towards a Micro-Economic Model for Resource Allocation in Grid Computing System. In Proceedings of the 2002 IEEE Canadian Conference on Electrical \& Computer Engineering (2002) 782-785

3. Foster, I., Kesselman, C.: The Globus project: a status report. Heterogeneous Computing Workshop, 1998. (HCW 98) Proceedings. (1998) 4 - 18

4. Buyya, R., Abramson, D., Giddy, J.: Nimrod-G: An Architecture for a Resource Management and Scheduling System in a Global Computational Grid. The 4th International Conference on High Performance Computing in Asia-Pacific Region (HPC Asia 2000), IEEE Computer Society Press, USA (2000)

5. Mandutianu, S.: Modeling Agent-Based Systems. Formal Approaches to Agent-Based Systems: In Proceedings of First International Workshop. FAABS 2000, Springer Verlag, LNCS Vol. 1871 (2001)

6. Nanda, S., Chiueh, T.: A Survey on Virtualization Technologies. RPE Report, (2005)

7. Zeigler, B.P. (ed.): The DEVS Environment for High-Performance Modeling and Simulation. IEEE CS \& E, Vol. 4, No3 (1997) 61-71 